February 2005 - NREL/CP-520-37440

\title{
Lattice-Mismatched Approaches for High-Performance, III-V Photovoltaic Energy Converters
}

M.W. Wanlass, S.P. Ahrenkiel, R.K. Ahrenkiel, D.S. Albin, J.J. Carapella, A. Duda, J.F. Geisz, S. Kurtz, and T. Moriarty

National Renewable Energy Laboratory

R.J. Wehrer and B. Wernsman Bechtel Bettis, Inc.

Prepared for the $31^{\text {st }}$ IEEE Photovoltaics Specialists Conference and Exhibition Lake Buena Vista, Florida Januarv 3-7, 2005
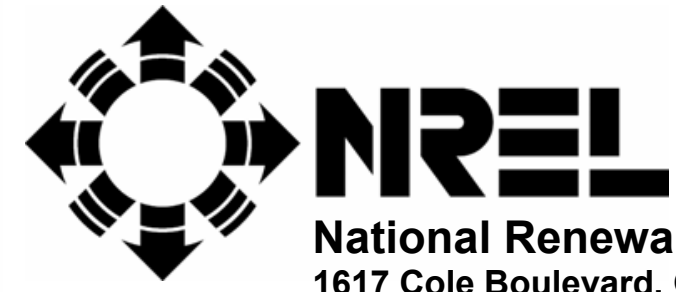

National Renewable Energy Laboratory 1617 Cole Boulevard, Golden, Colorado 80401-3393 303-275-3000 • www.nrel.gov

Operated for the U.S. Department of Energy Office of Energy Efficiency and Renewable Energy by Midwest Research Institute $\bullet$ Battelle 


\section{NOTICE}

The submitted manuscript has been offered by an employee of the Midwest Research Institute (MRI), a contractor of the US Government under Contract No. DE-AC36-99G010337. Accordingly, the US Government and MRI retain a nonexclusive royalty-free license to publish or reproduce the published form of this contribution, or allow others to do so, for US Government purposes.

This report was prepared as an account of work sponsored by an agency of the United States government. Neither the United States government nor any agency thereof, nor any of their employees, makes any warranty, express or implied, or assumes any legal liability or responsibility for the accuracy, completeness, or usefulness of any information, apparatus, product, or process disclosed, or represents that its use would not infringe privately owned rights. Reference herein to any specific commercial product, process, or service by trade name, trademark, manufacturer, or otherwise does not necessarily constitute or imply its endorsement, recommendation, or favoring by the United States government or any agency thereof. The views and opinions of authors expressed herein do not necessarily state or reflect those of the United States government or any agency thereof.

Available electronically at http://www.osti.gov/bridge

Available for a processing fee to U.S. Department of Energy and its contractors, in paper, from:

U.S. Department of Energy

Office of Scientific and Technical Information

P.O. Box 62

Oak Ridge, TN 37831-0062

phone: 865.576 .8401

fax: 865.576 .5728

email: mailto:reports@adonis.osti.gov

Available for sale to the public, in paper, from:

U.S. Department of Commerce

National Technical Information Service

5285 Port Royal Road

Springfield, VA 22161

phone: 800.553 .6847

fax: 703.605.6900

email: orders@ntis.fedworld.gov

online ordering: http://www.ntis.gov/ordering.htm 


\title{
LATTICE-MISMATCHED APPROACHES FOR HIGH-PERFORMANCE, III-V PHOTOVOLTAIC ENERGY CONVERTERS
}

\author{
M. W. Wanlass ${ }^{1}$, S. P. Ahrenkiel ${ }^{1}$, R. K. Ahrenkiel ${ }^{1}$, D. S. Albin ${ }^{1}$, J. J. Carapella ${ }^{1}$, A. Duda $^{1}$, J. F. Geisz ${ }^{1}$, Sarah Kurtz ${ }^{1}$, \\ T. Moriarty ${ }^{1}$, R. J. Wehrer ${ }^{2}$, and B. Wernsman ${ }^{2}$ \\ 1) National Renewable Energy Laboratory (NREL), 1617 Cole Boulevard, Golden, CO, USA \\ 2) Bechtel Bettis, Inc., 814 Pittsburgh-McKeesport Boulevard, West Mifflin, PA, USA
}

\begin{abstract}
We discuss lattice-mismatched (LMM) approaches utilizing compositionally step-graded layers and buffer layers that yield III-V photovoltaic devices with performance parameters equaling those of similar latticematched (LM) devices. Our progress in developing highperformance, LMM, InP-based GalnAs/InAsP materials and devices for thermophotovoltaic (TPV) energy conversion is highlighted. A novel, monolithic, multibandgap, tandem device for solar PV (SPV) conversion involving LMM materials is also presented along with promising preliminary performance results.
\end{abstract}

\section{INTRODUCTION}

The design options for III-V photovoltaic (PV) device structures based on LM heteroepitaxy are constrained due to the limited number of commercially available crystalline substrate materials. The design space is increased substantially by considering designs that involve LMM components. Traditionally, LMM approaches have been considered inferior due to the well-known associated problems such as high defect densities (dislocations and stacking faults), rough surface morphologies, epilayer cracking, and epiwafer bowing. At NREL, our goal is to make LMM heterostructures look and perform like LM ones (i.e., we strive to attain the highest Shockley-ReadHall lifetime, thinnest epistructure, and flattest epiwafer). In this paper, we discuss approaches utilizing compositionally step-graded layers and buffer layers that yield LMM PV devices with performance parameters equaling those of similar LM devices. Our progress in developing high-performance, LMM, InP-based GalnAs/InAsP materials and devices for thermophotovoltaic (TPV) energy conversion is discussed. Additionally, a novel, monolithic, multi-bandgap, tandem device for SPV conversion involving LMM materials is presented.

All of the test devices and PV converter structures discussed in this paper were grown using atmosphericpressure metalorganic vapor-phase epitaxy (MOVPE) in home-built systems at NREL. Trimethylindium, triethylgallium, trimethylgallium, trimethylaluminum, arsine, and phosphine were used as the primary reactants, with hydrogen sulfide, hydrogen selenide, disilane, carbon tetrachloride, and diethylzinc used as the doping precursors. Growth was performed at temperatures ranging from 600 to $700^{\circ} \mathrm{C}$ in a purified hydrogen ambient.

\section{LMM, InP-BASED THERMOPHOTOVOLTAIC ENERGY CONVERTER MATERIALS \& DEVICE DEVELOPMENT}

At NREL, we have been investigating low-bandgap, InP-based semiconductor alloys for TPV converter applications since 1991. The initial (LMM) device heterostructures utilized simple, mono-bandgap, shallowhomojunction devices and GalnAs compositionally graded regions to achieve the desired bandgaps ( 0.5-0.6 eV) on InP substrates [1]. The converter structures were subsequently improved in $1993 / 1994$ by employing two important design changes. Firstly, InAsP compositional step grades replaced GalnAs grades, as we found that the GalnAs material quality was improved. Secondly, GalnAs absorber layers were clad with InAsP layers to form double-heterostructure (DH) converters [2]. The InAsP cladding layers served to confine minority carriers to the GalnAs active layer, leading to a marked improvement in converter performance, both in improved carrier collection (i.e., increased quantum efficiency) and improved opencircuit voltage (i.e., reduced reverse-saturation current density). During the next ten years, several organizations became active in researching InP-based materials for TPV, and the work scope continued to expand to include the development of monolithic interconnected modules (MIMs) [3] and multi-bandgap, tandem converters [4, 5, 6, 7].

The main perceived disadvantage of the InP-based approach for TPV converters is that low-bandgap, GalnAs/InAsP DH converters are LMM with respect to InP. As mentioned earlier, LMM is known to introduce a wide range of problems in epistructures. Additionally, thick epistructures are often employed due to compositionally graded regions that are typically included to mitigate some of the aforementioned problems. The good news, as discussed later in this paper, is that many of the perceived problems have been addressed and, largely, solved. The InP-based approach is very well suited to the TPV problem; some of the important advantages have been described in a previous paper [8].

In developing LMM, InP-based materials for TPV, we have used a combination of x-ray diffraction (XRD), transmission electron microscopy (TEM), and 
cathodoluminescence $(\mathrm{CL})$ techniques to improve our understanding of the relaxation dynamics, and defect structure, in InAsP compositionally graded heterostructures and GalnAs/InAsP interfaces [9]. Additionally, we have employed resonant-cavity photoconductivity decay (RCPCD) [10] to characterize the minority-carrier lifetime as part of our qualification/optimization effort.

The low bandgaps that are typically required for TPV converters $(\sim 0.5-0.7 \mathrm{eV})$ dictate that LMM epistructures must be considered when growing GalnAs/InAsP heterostructures on $\mathrm{InP}$. Intermediate compositionally step-graded InAsP regions are included in the heterostructures to mitigate the deleterious effects of the LMM between the active GalnAs/InAsP $\mathrm{DH}$ and the $\operatorname{InP}$ substrate. Our studies have shown that all of the InAsP layers in a step grade, except the last one, which we refer to as the buffer layer, experience nearly complete relaxation. The high degree of relaxation in the step grade results in a flatter epiwafer. The InAsP buffer layer retains residual compressive strain, causing its in-plane lattice constant to be significantly smaller than the value for the fully relaxed condition. The reduction in lattice constant must be taken into account when growing a subsequent active layer of GalnAs in order to maintain a coherent GalnAs/InAsP interface. Otherwise, misfit dislocations will nucleate homogeneously at the interface, leading to a high interface recombination velocity and also, potentially, threading dislocations and stacking faults in the GalnAs active layer. Put simply, maintaining a coherent GalnAs/InAsP back interface is key to realizing a highquality $\mathrm{DH}$. We are also investigating a "displacement layer"concept [11] that involves including a layer between the buffer layer and the $\mathrm{DH}$. The displacement layer could take on different forms and functions, but its primary purpose is to improve the quality of the back interface in the $\mathrm{DH}$.

A significant amount of effort has been devoted to gaining an understanding of how the strain in the InAsP buffer layer varies with thickness. For typical thicknesses of $0.1-0.5 \mu \mathrm{m}$, we have observed that the buffer layer is strained significantly ( 0.20-0.25\%). We have also observed that the buffer layer strain is consistently higher than the equilibrium value according to theory [12]. The important point is that the buffer layer strain as a function of the buffer layer thickness is well characterized, and that it decreases in a monotonic fashion as the layer thickness increases.

The data in Fig. 1 were obtained from a set of structures where the unintentionally doped GalnAs (ntype, $\sim 5 \mathrm{E} 14 \mathrm{~cm}^{-3}$ ) active layer composition was held constant $\left(E_{g}=0.52 \mathrm{eV}, 2 \mu \mathrm{m}\right.$ thickness, a standard used for our lifetime measurements) while the number of compositional steps in the graded layer was varied (0.06 As mole fraction increment per step).

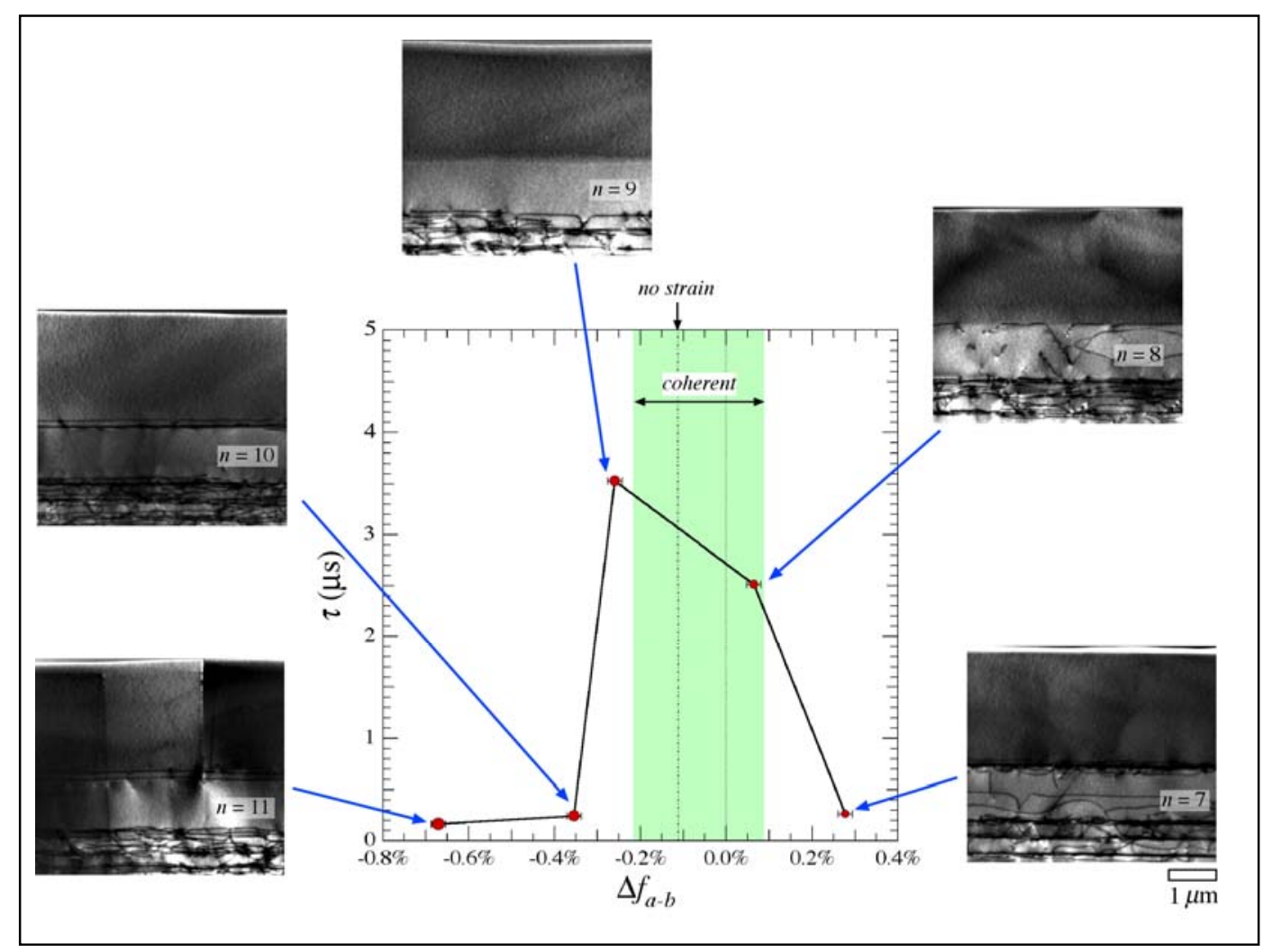

Figure 1. XTEM and RCPCD low-injection minority-carrier lifetime data for undoped, 0.52-eV GalnAs/lnAsP DHs grown on different InAsP buffer layers. The lifetime data are plotted as function of the differential lattice mismatch between the active layer and the buffer layer (calculated with respect to the InP substrate). 
Thus, the composition of the InAsP buffer layer was varied to put the GalnAs layer under different degrees of compression, and then tension. The region over which the active-layer/buffer-layer interface remains coherent (as determined empirically by XRD) is indicated. XTEM and RCPCD were used to correlate the quality of the back interface with the low-injection minority-carrier lifetime. Misfit and threading dislocations are readily seen for the cases outside of the coherent region, and the lifetimes are generally low. The high lifetimes correlate well with interface coherence.

High lifetimes, and commensurately large estimated diffusion lengths, have been obtained in our laboratory for both $\mathrm{n}$ - and p-type LMM DHs. Unintentionally doped ( $\left.5 \mathrm{E} 14 \mathrm{~cm}^{-3}\right), 0.5-\mathrm{eV}(1.7 \%$ LMM) n-DHs have low-injection hole lifetimes as high as $4.4 \mu \mathrm{s}$, as measured by RCPCD. The minority-carrier diffusion length was estimated (conservatively) by assuming that the mobility is half the value obtained for majority carriers experiencing the same background carrier density, and then using Einstein's relation, in conjunction with the relationship between the diffusion length, diffusivity, and lifetime, to calculate the diffusion length. Thus, for the n-DH above, the estimated hole diffusion length is $41 \mu \mathrm{m}$. A similar calculation was done for a 0.6-eV (1\% LMM), p-DH ( 1.5E16 $\mathrm{cm}^{-3}$, chosen so that recombination would be determined by radiative and $\mathrm{SRH}$ processes), which showed a $0.76 \mu \mathrm{s}$ low-injection electron lifetime. In this case, the estimated electron diffusion length is $126 \mu \mathrm{m}$. The above results indicate that the LMM DHs are of exceptional quality comparable to the best LM GalnAs/InP materials grown in our laboratory.

High-performance levels have also been demonstrated recently for 0.52-eV GalnAs/InAsP TPV converters, which have a relatively large LMM with respect to the $\operatorname{InP}$ substrate (1.6\%). Spectral quantum efficiency data for one of the better converters are given in Fig. 2 below.

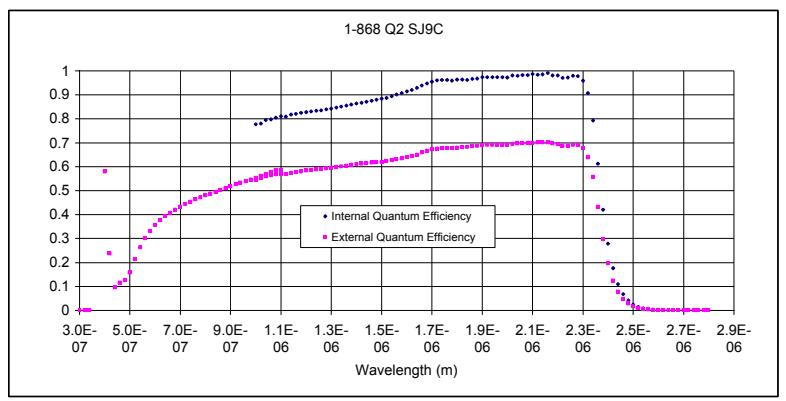

Figure 2. Spectral quantum efficiency data for a highperformance 0.52-eV GalnAs/InAsP TPV converter.

The data show near-unity internal quantum efficiency near the band edge, indicating that the material quality is excellent. It should be noted that the improved InAsP buffer-layer designs developed at NREL were used in these converter structures. The drop off in performance for wavelengths shorter than $1.7 \mu \mathrm{m}$ can be attributed to absorption in the InAsP window layer. Window layers with increased transparency should lead to improved performance. Photocurrent density versus voltage data for these devices, measured at $25^{\circ} \mathrm{C}$, have been analyzed, and a value of 1.07 for the ideality factor, and $39.6 \mu \mathrm{A} / \mathrm{cm}^{2}$ for the reverse-saturation current density, were determined. The above results are very encouraging as they indicate low recombination in the space-charge region. Also, the reverse-saturation current density value is less than a factor of two higher than that measured for the best 0.52-eV, LM GalnAsSb/GaSb converter [13].

\section{A NOVEL, ULTRA-THIN, MONOLITHIC, MULTI- BANDGAP, TANDEM SPV ENERGY CONVERTER}

Realistic modeling studies of series-connected, multibandgap, tandem solar cells show that the ideal bandgap for the bottom subcell in a triple-junction device is $\sim 1 \mathrm{eV}$. The above holds for a variety of potential tandem cell applications including those for space vehicle power and for terrestrial concentrators. Accordingly, a large effort has been devoted recently to the development of $\sim 1-e V, L M$ GalnAsN subcells for monolithic integration in Ge-based tandem structures that utilize GalnP $(\sim 1.85 \mathrm{eV})$ and GaAs $(\sim 1.4 \mathrm{eV})$ as the upper subcells. Unfortunately, so far, the quality of GalnAsN has been too poor to realize improved tandem performance through its inclusion. Thus, the need for a high-performance, monolithically integrable, 1-eV subcell still remains.

Here, we describe a novel approach for producing ultra-high-efficiency, monolithic, multi-bandgap, tandem (MMT) SPV converters that have improved performance, and other advantageous features, when compared with existing technologies. Accordingly, the objectives and key features of the approach are as follows:

1) To achieve SPV power conversion efficiencies of $35-40+\%$ for a variety of typical applications, the approach provides designs for MMT SPV power converters with optimal, or near-optimal, bandgaps for both space and terrestrial uses. Particularly, our approach provides a means of successfully combining the well-developed, medium- and high-bandgap, LM semiconductors grown on GaAs (or $\mathrm{Ge}$ ) substrates (e.g., GaAs, GalnAsP, AlGaAs, GalnP, and AIGalnP), with lower-bandgap ( 1 eV), LMM GalnAs alloys, in a monolithic structure. To date, ultrahigh-efficiency MMT SPV converters have not been demonstrated due to the lack of a suitable, highperformance, optimum, low-bandgap subcell option; the present approach provides a variety of schemes for including such an option.

2) To provide MMT SPV converter designs that can be grown epitaxially on $\mathrm{GaAs}$ or $\mathrm{Ge}$ substrates (monocrystalline or polycrystalline), which are thus compatible with current industrial techniques for producing SPV power generation systems. The design given here 
could also be grown on appropriate compliant or engineered substrates, if available.

3) To provide a method to fabricate ultra-thin MMT SPV converters that have the advantages described below:

a) For both terrestrial concentrator and space applications, ultra-thin devices provide the ultimate solution to thermal management, since the devices can be mounted on a robust, surrogate substrate (handle) of choice, and intimate contact with the heat sink can also be achieved. In contrast, devices processed on $\mathrm{Ge}$ or GaAs substrates have relatively poor thermal conductivity.

b) For space applications, the highest specific power $(\mathrm{W} / \mathrm{kg})$ is possible with handle-mounted, ultra-thin devices.

c) Ultra-thin MMT SPV converters can mounted on strong, flexible handles (e.g., metal foil or kapton), resulting in flexible devices, which may have specific advantages (e.g., PV system deployment in space).

d) The robust nature of handle-mounted, ultrathin devices is also a key advantage; devices processed on $\mathrm{Ge}$ or $\mathrm{GaAs}$ substates are relatively fragile.

e) Ultra-thin devices also open the possibility of reclaiming the scarce, expensive elements that make up the parent substrate (e.g., $\mathrm{Ga}$ in $\mathrm{GaAs}$ ).

Two key enabling concepts are used in our approach. The first is the use of inverted epistructures, which make possible the growth of the LM and LMM subcells in one growth run, on one side of the parent substrate, and in the proper optical sequence. With this scheme, the higherbandgap, LM subcells are grown first in the structure, thus possibly retaining their pristine (i.e., defect-free) quality. The second key feature is the use of transparent, compositionally step-graded layers, which allow the integration of LMM, infrared-responsive subcells (e.g., LMM GalnAs) into a monolithic scheme that includes LM higher-bandgap subcells. Inverted epistructures require handle mounting and parent substrate removal for efficient operation, thus, ultra-thin tandem device fabrication is a natural consequence of our approach.

Our initial work on the above-mentioned tandem cell approach has been focused on a series-connected, threesubcell device. The approach can be generalized to cover a wide range of designs; in a recent patent application, we discuss potential tandem device structures with two to six subcells [14]. The present device is grown on a GaAs substrate. LM n-GalnP\{ $(1 \mu \mathrm{m})$ etch-stop and n-GaAs $(0.4$ $\mu \mathrm{m})$ contact layers are first grown. A LM DH top subcell, consisting of an n-AllnP (25 nm) front-surface confinement layer (FSCL), n-GalnP (0.1 $\mu \mathrm{m}$, ordered) emitter layer, $\mathrm{p}$ GalnP (1.2 $\mu \mathrm{m}$, ordered) base layer, and a $\mathrm{p}$-GalnP $(0.1$ $\mu \mathrm{m}$, disordered) back-surface confinement layer (BSCL), is then deposited, followed by a $\mathrm{p}^{+} / \mathrm{n}^{+} \mathrm{GaAs}$ tunnel junction (24 $\mathrm{nm}$ total thickness). The LM middle subcell consists of an $n$-GalnP $(0.1 \mu \mathrm{m})$ emitter layer, $\mathrm{p}-\mathrm{GaAs}(2.5 \mu \mathrm{m})$ base layer, and a p-GalnP (50 nm, ordered) BSCL, followed by another $\mathrm{p}^{+} / \mathrm{n}^{+}$GaAs tunnel junction (24 $\mathrm{nm}$ total thickness). A transparent, compositionally step-graded GalnP layer is then grown to affect a change in lattice constant to match that of the low-bandgap ( $\sim \mathrm{eV}$ ) GalnAs alloy ( $\mathrm{Ga}$ mole fraction of 0.75 , LMM $\sim 2.2 \%$ ). The step grade contains 9 compositional steps with a Ga mole fraction increment of 0.03 per step, and a step thickness of $0.25 \mu \mathrm{m}$. The grade terminates with a $\mathrm{Ga}$ mole fraction of 0.24 in the GalnP buffer layer $(1 \mu \mathrm{m})$ to compensate for residual compressive strain (a $\mathrm{Ga}$ mole fraction of 0.26 in the GalnP buffer layer would be LM to the 1-eV GalnAs if both layers were fully relaxed). The LMM, 1-eV, DH subcell ( $n$ GalnP FSCL (50 nm), n-GalnAs emitter layer (150 nm), pGalnAs base layer $(2.9 \mu \mathrm{m})$, and $p$-GalnP BSCL $(50 \mathrm{~nm}))$ is then grown, along with a final $\mathrm{p}^{+}-$GalnAs $(0.1 \mu \mathrm{m})$ contact layer to facilitate processing. It should also be noted that a back-surface reflector (BSR) can be easily incorporated into the device at the top surface (which is actually the back of the device). The BSR can serve dual roles as both a sub-bandgap IR heat reflector for improved thermal management, and as a reflector that allows the LMM, 1-eV subcell to be grown half the usual thickness. The thinner LMM subcell takes less time to grow and should produce $\sim 20 \mathrm{mV}$ more output voltage due to the "narrow diode" effect (i.e., half the reverse-saturation current).

A general device processing sequence for inverted tandem cell structures is now described. The first step involves preparation of the top surface of the epistructure, which is actually the back surface of the device, by applying a back electrical contact, and, optionally, a backsurface reflector (BSR). The structure is than inverted and affixed to a pre-metallized "handle" material (in this case, conductive epoxy is used on an Al-coated Si wafer), which can be chosen to have a variety of advantageous properties and functions (e.g., strength, flexibilty, low cost, good thermal conductivity, selected electrical properties, etc.). The resultant handle-mounted device should have enhanced mechanical properties, and access to the back electrical contact should be established. The third step involves removal of the parent substrate and associated etch-stop layer (accomplished here using selective wetchemical etchants). In the final step, the top surface of the device has an electrical contact applied (gridded, electroplated $\mathrm{Au}$ ), and an antireflection coating (ARC) is also deposited. Additionally, the GaAs contact layer is selectively removed between the grids, and the active device layers are mesa isolated to define the device area and to eliminate electrical shunting. The ultra-thin tandem device is then complete and ready for operation.

We have performed semi-realistic modeling calculations, based on a rigorous approach for seriesconnected tandem subcells [15], to serve as a guide for the choice of the bottom subcell bandgap, and to predict potential performance, under operating conditions relevant to ultra-high performance devices. We assume that the bottom subcell quantum efficiency is 0.95 (spectrally independent) in all of the calculations. Also, the top and middle subcells are fixed to be GalnP $(1.87 \mathrm{eV})$ and $\mathrm{GaAs}$ $(1.42 \mathrm{eV})$, respectively. Results for the one-sun AM0 spectrum, at $25^{\circ} \mathrm{C}$, show that the optimum bottom subcell bandgap is $1.02 \mathrm{eV}$, with a tandem efficiency of $33.1 \%$. For terrestrial concentrator applications, we modeled for 
the low-AOD Direct spectrum at 250 suns concentration, $25^{\circ} \mathrm{C}$, and obtained an optimum bottom subcell bandgap of $1.01 \mathrm{eV}$, with a tandem efficiency of $41.5 \%$. The above results bode well for achieving the performance goals envisaged for ultra-high efficiency SPV converters.

In a preliminary effort, we have been successful in growing, processing, and testing monolithic, seriesconnected, handle-mounted, ultra-thin GalnP/GaAs/GalnAs tandem solar cells. Performance data for the best device fabricated to date are included in this section of the paper. Quantum efficiency (QE) and reflectance $(R)$ data are given in Fig. 3. The data generally show excellent carrier collection across a broad spectral range for all of the subcells. The $\mathrm{R}$ data, however, show that photocurrent gains are still possible at the far edges of the tandem response range. Improving the two-layer $\mathrm{ZnS} / \mathrm{MgF}_{2} \mathrm{ARC}$ will be a focus of future work. Interference effects are also observed in the QE data for the 1.02-eV bottom subcell, which occur because the subcell is optically thin with a BSR, causing it to behave like a FabryPerot cavity. The interference effects are also evident in the $\mathrm{R}$ data over the response range of the bottom subcell. It is important to note that the QE for the bottom subcell is excellent despite its $2.2 \%$ LMM with respect to the GaAs substrate.

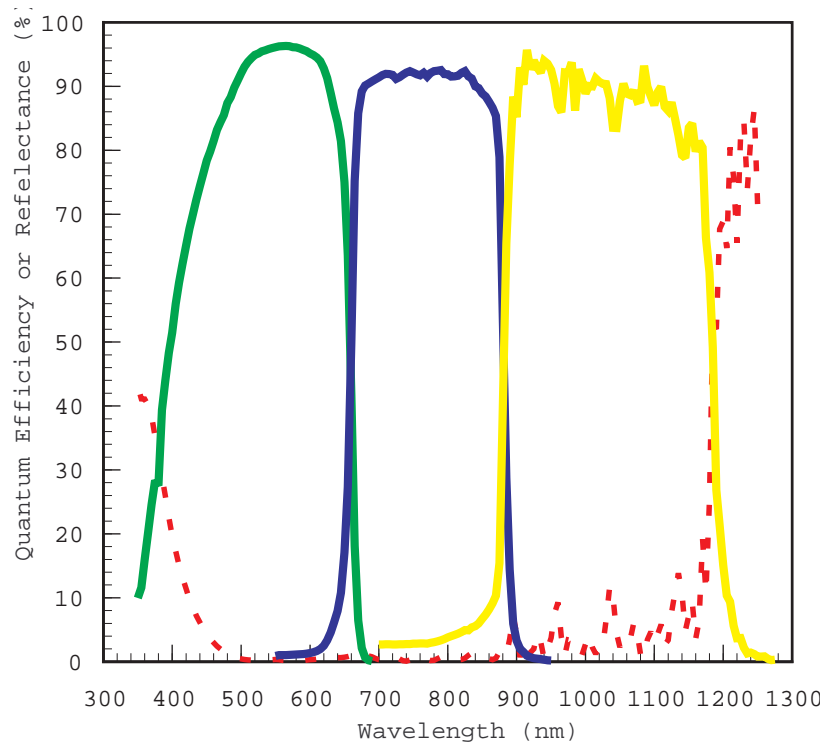

Figure 3. Composite spectral absolute external quantum efficiency (solid lines) and spectral reflectance (dotted line) data for an ultra-thin, handle-mounted GalnP/GaAs/GalnAs series-connected tandem solar cell.

Current-voltage data for the most efficient GalnP/GaAs/GalnAs tandem solar cell fabricated to date are shown in Fig. 4. The tandem cell is $31.1 \%$ efficient under the one-sun Global spectrum at $25^{\circ} \mathrm{C}$, which is only $\sim 1 \%$ absolute lower than the highest efficiency ever demonstrated for a solar cell under these conditions [16]. With continued development, we anticipate that these devices will be $34-35 \%$ efficient under the above conditions. Furthermore, versions of these devices tuned to perform optimally under both space (AM0, one sun), and terrestrial concentrator (low-AOD Direct, hundreds of suns), conditions are planned for near-term future work.

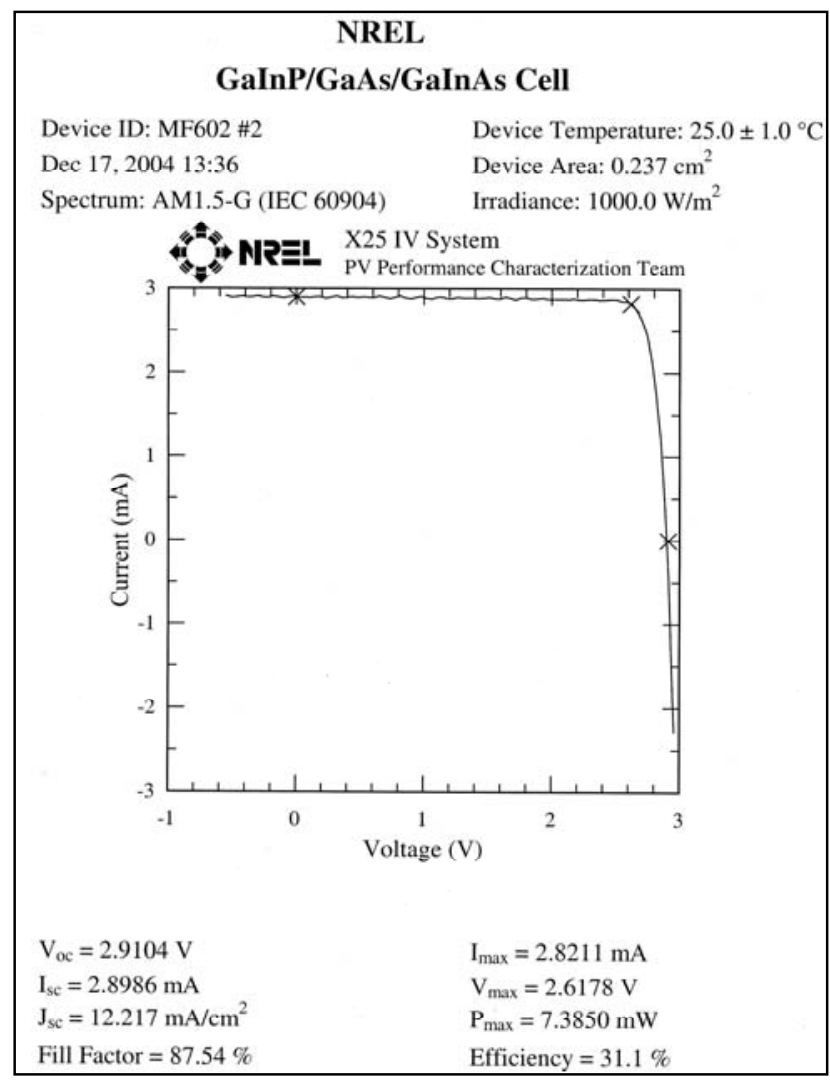

Figure 4. Current-Voltage data for a high-efficiency, ultrathin, handle-mounted GalnP/GaAs/GalnAs seriesconnected tandem solar cell measured under the one-sun Global spectrum at $25^{\circ} \mathrm{C}$.

\section{CONCLUSION}

The design space for epitaxial III-V PV device heterostructures is increased substantially by considering approaches that involve LMM components. Recent advances in the understanding of relaxation dynamics and heterointerface behavior in compositionally step-graded heterostructures with significant LMM $(\sim 2 \%)$ has resulted in the demonstration of LMM III-V materials and devices with properties equaling those of similar LM cases. Examples of highly successful applications of LMM approaches to both TPV and SPV devices have been discussed. LMM, InP-based TPV materials and devices have advanced considerably; GalnAs/InAsP DH devices with exceptional minority-carrier lifetimes and diffusion lengths have been demonstrated, along with 0.52-eV GalnAs/InAsP TPV converters with excellent performance characteristics. A novel, monolithic, ultra-thin GalnP/GaAs/GalnAs series-connected tandem solar cell, which uses a LMM, 1-eV bottom subcell, has been demonstrated with excellent performance. A conversion efficiency of $31.1 \%$ (one-sun Global spectrum, $25^{\circ} \mathrm{C}$ ) has been achieved for the SPV tandem in preliminary work, 
which is the second highest efficiency reported so far for any PV device under such operating conditions.

\section{ACKNOWLEDGEMENTS}

The authors gratefully acknowledge the support of both Bechtel Bettis, Inc., and the U. S. Department of Energy (under Contract DE-AC36-99G010337) for this work. We also thank Charlene Cramer and Michelle Young for assistance with the epitaxial growth and device processing, respectively, of the ultra-thin SPV tandem devices.

\section{REFERENCES}

[1] M. W. Wanlass et al., "Gax $\ln _{1-x} A s$ Thermophotovoltaic Converters," Sol. Energy Mater. Sol. Cells 41/42, 405 (1996).

[2] M. W. Wanlass, "Low-Bandgap, DoubleHeterostructure InAsP/GalnAs Photovoltaic Converter," U. S. Patent No. 6,300,557, 10/9/01.

[3] D. Wilt et al., "Monolithic Interconnected Modules (MIMs) for Thermophotovoltaic Energy Conversion," Semcond. Sci. Technol., vol. 18, pp. S209-S215, 2003.

[4] R. J. Wehrer et al., "0.74/0.55-eV Gax $\mathrm{In}_{1-}$ ${ }_{x}$ As/InAs ${ }_{y} P_{1-y}$ Monolithic, Tandem, MIM TPV Converters: Design, Growth, Processing and Performance," Proc. 29th IEEE Photovoltaic Spec. Conf., 5/20-24/02, New Orleans, LA, ISBN: 0-7803-7472-X, pp. 884-887, 2002.

[5] Rebecca Wehrer et al., "InGaAs SeriesConnected, Tandem, MIM TPV Converters," Proc. WCPEC-3 (30th IEEE PVSC), Osaka, Japan, May 15, 2003.

[6] S. L. Murray et al., "InGaAs Thermophotovoltaic Tandem Cell with Multi-Junction Interconnects on a Single Chip," Proc. 19th European Photovoltaic Solar Energy Conference and Exhibition, Paris, France, June 7-11, 2004.

[7] Mark W. Wanlass and Jeff Carapella, "LowBandgap, Monolithic, Multi-Bandgap Optoelectronic Devices," NREL IR \# 01-48, patent pending.

[8] M. W. Wanlass et al., "Recent Advances in LowBandgap, InP-Based GalnAs/InAsP Materials and Devices for Thermophotovoltaic (TPV) Energy Conversion," AIP Conf. Proc. 738, 427-435 (2004).

[9] S. P. Ahrenkiel et al., J. Electronic Mat. 33, 185 (2004).

[10] R. K. Ahrenkiel and S. W. Johnston, "An advanced technique for measuring minority-carrier parameters and defect properties of semiconductors," Materials Science and Engineering B102 (2003) 161-172.
[11] Mark W. Wanlass and S. Phillip Ahrenkiel, "Method for Optimizing Heteroepitaxial, LatticeMismatched Systems," NREL IR \# 02-01, patent pending.

[12] J. W. Matthews, in Epitaxial Growth (Part B), edited by J. W. Matthews (Academic Press, New York, 1975).

[13] Private communication with Dr. Michael Dashiell.

[14] M. W. Wanlass, "Approaches for Ultra-High Efficiency, Monolithic, Multi-Bandgap, Tandem Solar Photovoltaic Energy Converters," NREL IR \# 05-05, patent pending.

[15] M. W. Wanlass and D. S. Albin, "A Rigorous Analysis of Series-Connected, Multi-Bandgap, Tandem Thermophotovoltaic (TPV) Energy Converters," AIP Conf. Proc. 738, 462-470 (2004).

[16] Martin A. Green et al., "Solar Cell Efficiency Tables (Version 24)," Prog. Photovolt: Res. Appl. 2004; 12:365-372. 


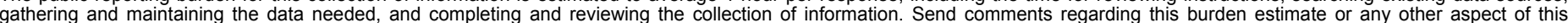

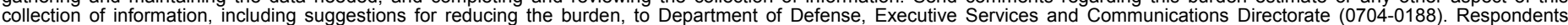

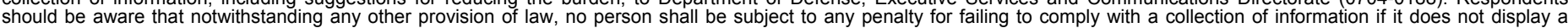

should be aware that notwithstand

PLEASE DO NOT RETURN YOUR FORM TO THE ABOVE ORGANIZATION.

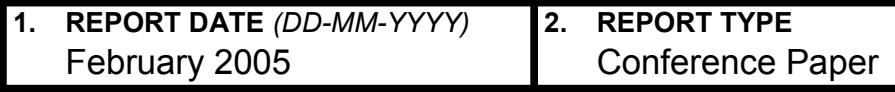

4. TITLE AND SUBTITLE

Lattice-Mismatched Approaches for High-Performance, III-V

Photovoltaic Energy Converters
3. DATES COVERED (From - To)

3-7 January 2005

5a. CONTRACT NUMBER

DE-AC36-99-GO10337

5b. GRANT NUMBER

5c. PROGRAM ELEMENT NUMBER

5d. PROJECT NUMBER

NREL/CP-520-37440

5e. TASK NUMBER

52005000

5f. WORK UNIT NUMBER

\section{PERFORMING ORGANIZATION NAME(S) AND ADDRESS(ES)}

National Renewable Energy Laboratory, 1617 Cole Blvd., Golden, CO

80401

Bechtel Bettis, Inc., 814 Pittsburgh-McKeesport Boulevard, West Mifflin, PA

9. SPONSORING/MONITORING AGENCY NAME(S) AND ADDRESS(ES)

8. PERFORMING ORGANIZATION REPORT NUMBER

NREL/CP-520-37440
10. SPONSOR/MONITOR'S ACRONYM(S)

NREL

11. SPONSORING/MONITORING AGENCY REPORT NUMBER

12. DISTRIBUTION AVAILABILITY STATEMENT

National Technical Information Service

U.S. Department of Commerce

5285 Port Royal Road

Springfield, VA 22161

13. SUPPLEMENTARY NOTES

\section{ABSTRACT (Maximum 200 Words)}

We discuss lattice-mismatched (LMM) approaches using compositionally step-graded layers and buffer layers that yield III-V photovoltaic devices with performance parameters equaling those of similar lattice-matched (LM) devices. Our progress in developing high-performance, LMM, InP-based GalnAs/InAsP materials and devices for thermophotovoltaic (TPV) energy conversion is highlighted. A novel, monolithic, multi-bandgap, tandem device for solar PV (SPV) conversion involving LMM materials is also presented, along with promising preliminary performance results.

\section{SUBJECT TERMS}

PV; lattice-mismatched (LMM); step-graded layers; buffer layers; lattice-matched (LM); devices; thermophotovoltaic (TPV); energy conversion;

\begin{tabular}{|c|c|c|}
\hline $\begin{array}{l}\text { a. REPORT } \\
\text { Unclassified }\end{array}$ & $\begin{array}{l}\text { b. ABSTRACT } \\
\text { Unclassified }\end{array}$ & $\begin{array}{l}\text { c. THIS PAGE } \\
\text { Unclassified }\end{array}$ \\
\hline
\end{tabular}

17. LIMITATION OF ABSTRACT

UL

18. NUMBER
OF PAGES

19a. NAME OF RESPONSIBLE PERSON

19b. TELEPHONE NUMBER (Include area code) 